\title{
The No Free Lunch Theorem and hypothesis of instinctive animal behavior
}

\author{
Alexey V. Melkikh * \\ Ural Federal University, Yekaterinburg, Russia
}

Received: September 14, 2014

Accepted: October 12, $2014 \quad$ Online Published: October 24, 2014

DOI: $10.5430 /$ air.v3n4p43

URL: http://dx.doi.org/10.5430/air.v3n4p43

\begin{abstract}
The problem of knowledge acquisition in animals is considered from the point of view of cybernetics. We show that all types of animal behavior can be consistently explained on the basis of innate behavior programs and the creation of new behavior programs is logically inconsistent. The hypothesis that all animal behavior is completely innate is proposed. As a possible physical implementation of the storage of congenital programs, we considered quantum entanglement of biologically important molecules. Experiments to test hypotheses are proposed.
\end{abstract}

Key Words: Learning, Knowledge acquisition, No Free Lunch Theorem, Innate programs of behavior, Recognition

\section{Introduction}

Researchers believe that one part of the behavior of organisms is innate and another part appears due to learning. As a result of learning, animals acquire the knowledge necessary to survive in a changing environment. However, the mechanism of the learning process is still far from clear.

We consider the following issues: the cause of the process of acquiring of knowledge; the programs for this process; what these programs should include; the "ability to learn"; whether this is a universal ability; and whether this ability can be formalized.

To answer these questions, we require a precise definition of the terms that describe animal behavior. However, many of the terms associated with behavior are not well defined. Understanding the phenotypic plasticity of behavior led us to new terms, which reflect the influence of the environment on genes. However, these terms are also not well defined, and the mechanism of plasticity is unclear.
However, many forms of behavior implicitly assume a priori information on the analyzed object. For example, when considering and modeling decision-making in animals, researchers implicitly assume that the images that surround the animal are recognized. However, a new situation may be not recognized. In this case, an adequate decision cannot be made. For example, when fruit flies learn of some smells, ${ }^{[1]}$ researchers assume that the smells are registered by receptors. However, registration and recognition are significantly different operations. If the smell is registered and recognized, some types of a priori programs can run in an adequate given situation. However, if the smell is registered by receptors, but not recognized (for example, the smell of a new synthetic material), then we ask whether there are any new programs adequate for the new situation.

With regard to conditioned reflexes, another question arises when a stimulus accompanies a famous image. An animal can fix this stimulus (e.g., its repetition over time), find regularities in its appearance (the task of clustering, which can be performed without comparison to the standard), and then

*Correspondence: Alexey V. Melkikh; Email: melkikh2008@ rambler.ru; Address: Yekaterinburg, 620002, Mira str. 19. Russia. 
use this information as a switch for the implementation of various aprioristic programs of behavior (i.e., to implement the program or not). The question is whether a system can create new programs in this case. We ask if a system can form a new behavior in response to an unknown stimulus.

The problem of knowledge acquisition in animals is at the intersection of multiple sciences. To answer these questions, first, we are to use data from modern cognitive and biological science of behavior and genes. Second, we are to use mathematics based on proofs and the achievements of cybernetics.

One issue is that cybernetics (information theory, control theory, optimization methods, etc.) is often used to solve several other problems, which are not focused on the properties of specific organisms. This issue is seen, for example, when considering the concept of learning in terms of mathematics and technical systems or in terms of the behavioral sciences. The underlying concepts and the axiomatic system are significantly different. This issue complicates the use of proof-based mathematics in real living systems. The results of interpenetration (symbiosis) of the behavioral sciences and cybernetics are results of fundamental importance for understanding the behavior of living and technical systems.

Cybernetics historically arose as an attempt to unify mathematical descriptions of behavior in living and non-living systems. ${ }^{[2]}$ It includes the study of feedback, black boxes and derived concepts, such as control and communication in living organisms, machines and organizations. It focuses on how to accomplish something (digital, mechanical or biological), processes information that reacts to it and may be modified or changed. One of the key concepts of cybernetics that can be considered is the notion of optimality. The behavior of both living and technical systems is committed to optimal (in some sense). The study of cybernetics consists of figuring out what it is, to what extent and why it is optimal for a wide class of systems.

Despite the fact that a number of attempts were made to use system descriptions of living organisms, ${ }^{[2-4]}$ there is now a need for a systems approach to behavior (e.g., in the framework of systems biology). This approach can be seen as a new synthesis in the behavioral sciences. This new synthesis must be based on the data of modern behavioral genetics and phenotypic plasticity. This article is an attempt (based on a systems approach) to answer the following question: does the organism contain all of the programs for its future behavior after the individual development, or do the programs arise (modified) as a result of learning and interaction with the environment?

\section{Learning, adaptation and knowledge ac- quisition}

We consider the relationship between the concepts of "learning", "adaptation" and "acquisition of knowledge" in living and technical systems.

We note that part of the behavior of animals is definitely innate, implemented without learning. However, this raises the following question: what behavior is innate? The answer is not so simple because, as noted in Ref.5 difficulties arise during experimental determination of innate behavior.

Another part of behavior is associated with learning. There are many definitions of learning. We present some of these definitions.

Thorpe ${ }^{[6]}$ defined learning as "a process consisting in the appearance of adaptive changes in individual behavior as a result of the acquisition of experience." Shettleworth ${ }^{[5]}$ defined learning as "earning, or equivalently memory, is a change in state due to experience" but noted, however, that this definition includes many nuances.

Because many of the definitions of "learning" are related to the concept of "adaptation", the latter term also needs to be defined.

The concept of adaptation has many meanings both in terms of technical and living systems. We can state that all animal behavior is adaptive to a varying degree, i.e., aimed at maximizing a "gain" by the organism. For example, this gain can be represented, with other aspects being equal, in the form of fastest goal achievement or the least cost of any action, among others. In biological systems, adaptation means adaptation of an organism to external conditions during evolution, including morphological and behavioral components. Adaptation can ensure survival in a particular habitat, resistance to abiotic and biological factors, and success in competition with other species, populations and individuals. The adaptive response of the organism is manifested at the organ and tissue level, the behavioral level of the organism and the population evolution level.

Subsequent modeling of artificial living systems followed the path of building more adequate models of such systems with a more detailed modeling of their interactions with the environment. The special term "animat" was defined, which is an artificial organism capable of adapting to the environment. A characteristic feature of adaptive behavior in the modeling of different motion mechanisms is executed by the effectors of different actions and the particular architecture of the block of information processing and decision making. ${ }^{[7-11]}$

Thus, adaptation is a general concept (which includes any function of the organism, including homeostatic), but this concept is not well suited to the problem in this paper because of its vagueness and imprecise definition. 
We now turn to the classification of animal learning. There are several classifications of animal learning. For example, according to Ref.12, learning can be identified as habituation, associative learning (conditioned reflexes of the first type, trials and errors and the conditioned reflexes of the second type), latent learning, insight-learning, or imprinting.

According to Ref.13, simple forms of learning that do not require the formation of concepts are habituation, sensitization and classical (Pavlov) conditioning.

The different definitions and classifications of animal learning raise the following questions:

What changes occur in the organism (neurons, genes) during these types of learning? Does the organism really receive new information as a result of learning, or do only existing innate programs of behavior run during this process?

One of the problems arising in the study of animal behavior is that changes to the animal that occur during the process of learning are not always easy to register. Therefore comparing the properties of learning in such systems when the device is fully known is useful. These are the properties of the technical system. We consider several definitions of learning in terms of artificial intelligence.

Herbert Simon defined "learning" as "any change in a system, that allows it to perform better the second time on repetition of the same task or on another task drawn from the same population". [14]

Computational learning theory focuses on the design and analysis of algorithms for making predictions about the future based on past experiences. ${ }^{[15]}$

The idea behind designing a learning system is to guarantee robust behavior without complete knowledge, if any, of the system/environment to be controlled. A crucial advantage of reinforcement learning compared to other learning approaches is that it requires no information about the environment except for the reinforcement signal. ${ }^{[16,17]}$

Learning is defined as any permanent change in behavior as a result of past experience, and a learning system should therefore have the ability to improve its behavior with time, toward a final goal. In a purely mathematical context, the goal of a learning system is the optimization of a functional not known explicitly. ${ }^{[18]}$

There are some classes and models of learning. [19]

These definitions of learning have very specific advantages for certain tasks and also possess a number of drawbacks. First, these definitions for systems with invariable internal structure and for systems whose structure can be changed at will, must be different. If, for example, a man interferes directly and actually creates another system instead of an existing system, is this learning? Such a process obviously has nothing to do with learning, but is related to another domain. Therefore, we need to elaborate the types of interaction between an intelligent system and the environment.

Second, we do not know whether the ability of a system to acquire new knowledge is considered learning or whether the system always operates within a priori rules and the commands given to it. Clearly, some types of learning are not related to the acquisition of new knowledge and represent only a selection of system software from an a priori existing list.

We will distinguish the concepts of "learning" and "knowledge acquisition". First, we define the difference between these concepts at an intuitive level and then further define them more rigorously mathematically. Indeed, the acquisition of knowledge means more than just learning. Typically, the acquisition of knowledge is associated only with humans and some higher animals. Learning is a broader concept inherent to both living and artificial systems. Learning is a broader concept that is inherent to both living and artificial systems. Knowledge can be defined as familiarity with someone or something, which can include facts, information, descriptions, or skills acquired through experience or education. It can refer to the theoretical or practical understanding of a subject.

We associate learning with the process of adapting the system to the environment in the presence of a priori knowledge (useful information) of the environment. We associate acquisition of knowledge with the process, following the emergence of an unrecognized environmental image. This concept does not include preparation of the system and is related only to giving the system information about the external environment through receptors, which results in the system behavior becoming more adequate. A system that acquires knowledge should behave as if adjusting to a certain environment based on specific algorithms.

We associate higher forms of learning (behavior) with the acquisition of knowledge. We associate learning with adaptation based on a priori knowledge without receiving new knowledge. A physical realization of a priori knowledge includes a priori programs that exist in the system prior to its learning. With regard to animals, these programs are innate behavior programs.

All modern systems of artificial intelligence are capable of learning only in the presence of aprioristic knowledge (useful information).

(1) Heuristic methods of problem solving are based on aprioristic information about the object domain that comprises the problem. The heuristics may prove to be incapable of finding the solution altogether. This limitation cannot be removed even in the best heuris- 
tics. ${ }^{[20]}$ The key question is how the heuristics arise. The theory does not answer the question of how we can develop a new heuristic.

(2) The recursive search represents a natural method for realization of such strategies of artificial intelligence as the graph search. However, an exact objective should be set for this method to be used. If objects (even one object) are not defined, the recursive procedure cannot be applied. ${ }^{[21]}$

(3) Production systems are used for conflict resolution. In this case, the system requires an a priori standard sample (pattern), which determines the possibility of using the rules of production systems. ${ }^{[22]}$

(4) Expert systems rely on the same rules. ${ }^{[21]}$ The core of an expert system is a knowledge base, which contains knowledge from a particular applied domain. Knowledge in an object domain determines and updates an expert database. ${ }^{[23]}$ Can we possibly imagine a system that acts as an expert for itself? This is obviously not possible because a code can be corrected only when correct knowledge is known.

(5) Learning of neural networks ${ }^{[21]}$ is based on the presentation of a priori preset training examples. Indeed, learning of a neural network (with a teacher) implies the assignment of relation weights between neurons, which is realized in a special regime (but not in the pattern recognition regime). In this case, the person who assigns standard templates to this neural network has aprioristic information about the object. In the case of unsupervised learning, neural networks are only capable of clustering information. In this case, there is no recognition. As shown below, this mode relates to conditioned reflexes. Another problem is how to determine that the system has come to the correct decision.

(6) Recently, hybrid connectionist-symbolic systems based on a synthesis of neural networks and symbolic representation of knowledge ${ }^{[24-26]}$ were proposed. These systems help in the knowledge acquisition in a variety of applications. However, the term "knowledge acquisition" in such a system is used in a different sense than in this article. In the frame of this approach, "knowledge acquisition" means that with help of this knowledge some useful task was solved (for example, in medicine). Functioning of such systems a priori assumes that all characters (or simple signals) or the words, written in a specific language, are recognized by human or computer. In this sense, they are innate. Wherein the problem of determining whether the knowledge are innate to humans is not considered.

Can an organism (a system) update the knowledge base itself and adapt to unforeseen circumstances? Brachman ${ }^{[27]}$ argues that there are systems that "know what they're do- ing". However, the scheme of such a system is based on neural network learning, and the new qualities such a system has compared with neural networks are unclear.

The problem of classification of new concepts based on a Bayesian classifier is discussed in an article. ${ }^{[28]}$ Learning is considered to be a hierarchical process in which the unrecognized object can be extracted into a separate class. However, the paper does not consider further actions with this object. In this context, as is evident, this classification is not knowledge acquisition because acquiring knowledge requires the appropriate action in relation to this new object.

Article $^{[29]}$ correctly states that the theory of knowledge is required. On the one hand, epistemology, which is the branch of philosophy concerned with the nature and scope of knowledge, exists; it is also referred to as the "theory of knowledge". It questions what knowledge is, how it can be acquired, and the extent to which knowledge pertinent to any given subject or entity can be acquired. On the other hand, we need the mathematical theory of knowledge, which includes objective criteria and modeling. Researchers assume that such a theory can be established on the basis of mathematical logic. We note the importance of the concept of information entropy with respect to the assessment of the information contained in various statements. We note that, in this connection, mathematical logic only works with certain concepts. In this logic, the acquisition of new mechanisms was not included. The relation between information and knowledge will be discussed below (see 5.1).

Often, the acquisition of knowledge is associated with the concept of "intelligence". There are many definitions of "intelligence". For example, Gottfredson ${ }^{[30]}$ defines this concept:

A very general mental capability that, among other things, involves the ability to reason, plan, solve problems, think abstractly, comprehend complex ideas, learn quickly and learn from experience. It is not merely book learning, a narrow academic skill, or test-taking smarts. Rather, it reflects a broader and deeper capability for comprehending our surroundings--catching on," "making sense" of things, or "figuring out" what to do.

Article $^{[31]}$ notes that intelligence is difficult to define, but we can select intelligence formally based on the information with which it works. Intelligence is a feature for upgrading information with higher-level knowledge (knowledge about knowledge). However, if the knowledge of knowledge exists in the system a priori, then this secondary knowledge is not new, but is derived based on the laws of logic. The alternative is that the primary knowledge was not complete (that is, there is only partial knowledge about any subject that could somehow be replenished). However, in this case, 
the way secondary knowledge is obtained is unclear.

Thus, the mechanisms of research, upgrading, and granulation of knowledge are not fully defined despite awareness of the need to examine them. Can we possibly write an a priori computer program that realizes all of these actions?

Another important aspect of learning, the creation of new behavior programs (in addition to the already existing a priori or innate programs), is needed to more adequately respond to the recognized situation. This aspect could occur by either logical analysis or any interaction with the environment (the trial and error method).

Therefore, we combine these two aspects in the term "knowledge acquisition": the creation of new behavior programs for known or new environments, which allows the system to behave more appropriately. Thus, the review of the concepts of learning, adaptation and knowledge acquisition in living and technical systems allows us to reach the following conclusions:

(1) Classification of learning is useful in a number of applications, but does not answer some fundamental questions. Classification of learning and behavior needs to be based on more precise definitions, such as pattern recognition and a priori information.

(2) Part of animal behavior is innate and performed on the basis of innate behavior programs. In technical systems, this corresponds to a priori programs that are initialized prior to the start of the learning process.

(3) The higher forms of animal and human learning naturally associate with the term "acquisition of knowledge." However, the mechanism of knowledge acquisition remains unclear. In particular, the adaptation of the organism to the new environment is unclear.

The last conclusion is a principal idea in this article and will be discussed in section 4 . We consider the basic forms of learning in terms of recognition tasks so that we can understand which of them only need innate behavior programs and which cannot be reduced to such programs.

\section{Pavlov conditioning, plasticity and learn- ing}

\subsection{Pavlov conditioning and the task of clustering}

As already noted above, some types of simple learning can be attributed to habituation, sensitization and classical (Pavlov) conditioning. These elementary forms are found in most animals, including those with simple nervous systems. Pavlovian conditioning ${ }^{[32]}$ is a form of learning in which the conditioned stimulus comes to signal the occurrence of a second stimulus known as the unconditioned stimulus. A stimulus is a factor that causes a response in an organism. The conditioned response is the learned response to the previously neutral stimulus. The unconditioned stimulus is usually a biologically significant stimulus, such as food or pain, that elicits a response from the start. The conditioned stimulus usually produces no particular response at first, but it elicits the conditioned response after conditioning. Classical conditioning differs from operant or instrumental conditioning, in which behavior emitted by the subject is strengthened or weakened by its consequences (i.e., reward or punishment). ${ }^{[3]}$ We show that from the point of view of cybernetics, such forms correspond to the problem of clustering (cluster analysis), which does not require the recognition of a new object. Innate programs are sufficient for its solution.

Extensive literature is devoted to Pavlovian conditioning, and research in this field is ongoing (see, e.g., the RescorlaWagner model ${ }^{[34,35]}$ ). A distinctive feature of the Pavlovian response is that the external stimulus, which produces the conditioned reflex, may not be recognized by the animals. Indeed, many of the stimuli ${ }^{[51]}$ are artificial signals that animals do not find in nature. However, the formation of a conditioned reflex only requires the signal to be registered by the receptors of the animal and stored in memory. In this case, the reflex is to find regularities in the time (spatial) appearance of objects.

Obviously, from the point of view of cybernetics, Pavlovian conditioning corresponds to the problem of clustering. Cluster analysis is the problem of partitioning a given sample of objects (cases) into subsets called clusters. Each cluster is composed of similar objects, and objects of different clusters are significantly different. Clustering refers to the task of statistical analysis, as well as to a wide class of unsupervised learning problems. There are many clustering methods used in different areas (for example ${ }^{[36-39]}$ ).

The problem of recognition (classification) is different from the problem of clustering. One of the important stages of the recognition problem is comparing the observed object with a priori defined standards.

The classic statement of the problem of pattern recognition is a given set of objects that need to be classified, i.e. each object needs to be assigned to a class. Information about the classes is presented a priori (there is a set of objects - sample - the class membership of which is known). In neural networks, this problem corresponds to learning with a teacher; membership of certain images (standards) to the classes is determined a priori.

Thus, the fundamental difference between the problem of clustering and the problem of recognition (classification) is that the problem of clustering only pertains to the proximity between objects under investigation (in some sense); the analyzed objects are not known. The system cannot make an adequate decision concerning an object.

The elaboration of a conditioned reflex with respect to the 
unrecognized image is possible because the animal needs a proper response to the primary stimulus, but not to this new stimulus. This stimulus is recognized, and a reaction to it is innate.

This basic difference is connected by a paradox, which will be reviewed in section 4.1 .

Thus, the implementation of the Pavlovian reflexes requires innate behavior programs.

Habituation and sensitization can be represented as special cases of conditioned reflexes. For these programs, innate programs are sufficient. Besides the response to a stimulus, an innate program includes a condition such as "if the stimulus is repeated $n$ times, do not respond to it." The origin of this condition is obvious; the origin is due to the cost decrease in cases in which the reaction is not necessary.

What happens in the new environment? Will the organism only confuse the new environment with an old environment? Can the animal develop new programs for this environment?

\subsection{Plasticity and learning}

There are two prior opposing theories linking behavior and genotype. In one theory conditionally called "behaviorism", the important role of genes was denied. However, there was another trend ("genetic determinism") that stated that genes completely determine behavior.

At present, these extreme views have not been confirmed. The study of the individual development of an organism shows that in different environments, the same genotype may be expressed differently. The conclusion is that the phenotype and behavior of an animal depend not only on genes but also on the environment. ${ }^{[40,41]}$

However, as shown by experiments, identifying the genes responsible for specific aspects of behavior (e.g., learning) is difficult. For example, Glanzman ${ }^{[42]}$ notes that the presence of molecular pathways is not sufficient to understand learning. Examples of Aplisia and Drosophila behavior showed that molecules such as GABA and glutamate are associated with neuronal plasticity. However, by themselves, molecules cannot provide a detailed explanation of behavior without consideration of neural networks. "The take-home lesson from the two studies discussed here is that knowledge of the key molecular players does not provide a short cut to understanding memory and cognition". ${ }^{42]}$ This conclusion can be attributed to other organisms and behaviors.

Thus, the organism can change its phenotype in response to changes in the environment (phenotypic plasticity). If we consider behavior, the special case of this phenotypic plasticity can be considered as neuroplasticity, changes in connections between neurons as well as the strength of synaptic contacts (synaptic plasticity) under the influence of experience. Many of the mechanisms of synaptic plasticity are common in vertebrates and invertebrates. ${ }^{[42]}$

Noble ${ }^{[43]}$ examined the relationship between different levels of causality in the cell. In his view, the genes do not represent a program because many properties of the organism (cells) can be realized only at the higher systems level.

Questions about the relationship between genes and behavior (in particular, the acquisition of knowledge) are the principal problems addressed in this article. To what extent can we speak about the behavior program in relation to the animal? We consider these questions in more detail.

We first discuss the question of what can be called a program. Of course, in the context of this problem, we are not considering a program written in a computer language. The term "program" should be understood to be any sequence of actions, written in any language, that with the given initial data and other things being equal, the external conditions lead to the same result. We must keep in mind that calculation errors are a natural consequence of any program (computer or based on other principles of calculations). In this sense, any physical process described by the equations, e.g., based on the conservation laws, can be considered by the program. We also emphasize that the program need not be linear (the flow of information goes in one direction only from the input to the result). In many cases (living and technical systems), there are complex program structures, and under certain conditions, the programs can run in a completely different way. To understand the program as a mathematical expression pattern, we use the principle of determinism as a basis for modeling systems.

The principle of determinism in this case can be understood to be a regular behavior for the same external conditions. This natural behavior occurs in animals (including the case of learning). In fact, this natural behavior simply coincides with the scientific method because the basis of science is repeatability (within errors) of experimental results.

In this sense, the plasticity of neural systems, phenotype and behavior means only one thing: there must be a regularity of system behavior at the molecular level. Under the same external conditions and neuronal states, certain connections between adjacent neurons, strengthening of certain synapses, specific phenotype, and behavior, among others, are formed.

Data on neuroplasticity (synaptic plasticity) are often interpreted to mean that the synaptic contacts and, therefore, the entire nervous system should be able to adapt to arbitrary situations. The plasticity acts in some sense as a basis for receiving knowledge about the environment. However, this statement is incorrect because obtaining information from the environment at the stage of recognition (at the molecular level or above) is still inevitable. If the neuron (or a set of them) has no a priori molecular response to a certain reaction, then the answer that follows is not necessarily ap- 
propriate. Moreover, the presence of an unknown substance (or just a new conformation of a known substance) is likely to lead the network of molecular reactions to chaos.

Thus, the plasticity of the organism in all its forms (phenotypic, synaptic, etc.) is the least complicated but, nevertheless, is also the set of aprioristic programs. Only in this way can the system provide an adequate response to an external signal.

Another question here is how much of this information is needed for the formation of such a complex plasticity and where the information originates. We must separate two essentially different processes: morphogenesis (ontogenesis) and learning. We will consider the second process, meaning that the organism is already formed. Consideration of the mechanisms of morphogenesis (ontogenesis) is beyond the scope of this article.

Another objection to the analogy between the brain and a computer program is a famous thought experiment by Searle, the Chinese Room. Searle ${ }^{[44]}$ argues that the brain's mind (especially the human mind) is not a computer program because a computer does not understand what is it doing, but the brain does. If some instructions that we receive are written in a language foreign to us, then we do not understand what we are doing if we run them. However, such a statement is incorrect because it implicitly assumes that the computer does not recognize the images but only clusters them (see above). In this sense, the "understanding" (for living or technical systems) does not require anything other than recognition. This operation can certainly be performed by a computer. If we consider a living system or a computer in terms of language, the "understanding" of a word corresponds to the notion of "semantics." However, for the internal computer language, it is clear that all words have recognized meanings. For each word, certain action is permissible. Such a language exists at all levels of biological organization, including the molecular level. ${ }^{[45]}$

In general (especially for a man), "understanding" is a more general term than "recognition", because the analyzed object is always in some context (cultural, historical, etc.). However, if we consider the "understanding" in the narrower sense of the word (understanding leads to an adequate solution of the problem), in this approximation we can consider "understanding" and "recognition" as the same term. Thus, both the processes of recognition ("understanding") and clustering can occur in living and technical systems that are based on computer programs.

The relationship between the brain and mind (mind-body problem) is an unsolved question. A mind is the set of cognitive faculties that enables consciousness, perception, thinking, judgement, and memory - a characteristic of humans, but which also may apply to other life forms. Whether the mind is somehow separate from physical existence, deriving from and reducible to physical phenomena, such as neurological processes, or whether the mind is identical with the brain or some activity of the brain? The mind-body problem in philosophy examines the relationship between mind and matter, and, in particular, the relationship between consciousness and the brain.

Because changes within the organism and its interactions with the environment are crucial when considering knowledge acquisition and learning, these interactions should be defined more strictly. For the processes of knowledge acquisition and learning, the interaction of the organism and environment must be performed only through recognition. The organism obtains all the information about the environment through its senses. Furthermore, this information is subject to recognition, after which the organism decides what to do in the current situation. However, in principle, an organism can interact with the environment in another way - preparation. The structure of the system can be directly changed by acting on it from the outside (not through the senses). This method is widely used in engineering systems: for example, a computer user has the ability to replace any part of a system, which creates a different system. However, this method is not related to learning and knowledge acquisition (as in this case another system learns); in addition, this method does not occur in living systems. Thus, the preparation of the system will not be considered further.

As a rule, the ability of animals and humans to form concepts, generalize, and adapt to arbitrary environments, among others, are ascribed to higher forms of learning. These properties are often associated with the concept of "intelligence." Researchers believe that animals and human beings are capable of this type of learning.

We associate higher forms of behavior to the acquisition of knowledge (see above). We assume that for such behavior, innate programs are not sufficient. In this case, a more rigorous mathematical treatment of this behavior will be given below.

Thus, accounting for what we discussed about the learning of animals and the behavior of artificial intelligent systems, we can distinguish two classes of behavior (see Table 1):

Table 1: Two classes of behavior

\begin{tabular}{|c|c|}
\hline $\begin{array}{l}\text { Behavior based on innate } \\
\text { programs (A) }\end{array}$ & $\begin{array}{l}\text { Behavior with knowledge } \\
\text { acquisition (B) }\end{array}$ \\
\hline $\begin{array}{l}\text { Includes conditional and } \\
\text { unconditional reflexes, as well as } \\
\text { the simplest forms of learning }\end{array}$ & $\begin{array}{l}\text { Includes learning experiences } \\
\text { (interactions with other animals), as } \\
\text { well as the logical conclusion of } \\
\text { new concepts }\end{array}$ \\
\hline $\begin{array}{l}\text { An animal (Intelligent system) } \\
\text { meets a recognized object. This is } \\
\text { accompanied by the running of } \\
\text { innate programs. An unrecog- } \\
\text { nized object can only trigger } \\
\text { innate programs. An adequate } \\
\text { response to the unrecognized } \\
\text { object is impossible. }\end{array}$ & $\begin{array}{l}\text { An animal (Intelligent system) } \\
\text { meets an unrecognized object and } \\
\text { begins to respond to it adequately } \\
\text { as a result of the acquisition of } \\
\text { knowledge. }\end{array}$ \\
\hline
\end{tabular}


Let us consider a formal mathematical model of the behavior of type B and show that this behavior is either reduced to A or is logically inconsistent.

\section{The knowledge acquisition paradox and hypothesis on instinctive behavior}

\subsection{The knowledge acquisition paradox and No Free Lunch Theorem}

We formally consider the situation in which the organism meets a new unrecognized object and show that adequate behavior in this case (type B behavior) is contradictory.

The object in the field of view of the organism (artificial systems) is not recognized. Therefore, for an adequate response to this situation, the organism (system) should have algorithms to adequately respond to the uncertainty of a situation in advance. There must be an algorithm that is optimal (in a certain a priori sense) for all tasks.

However, the No Free Lunch Theorem claims that such an algorithm is impossible. During the 1990s, the No Free Lunch Theorems for the processes of search and optimization were proven. ${ }^{[46]}$ The essence of the No Free Lunch Theorem is that there can be no universal algorithm that is effective for all functions.

The optimization problem is the basis of any decision making task. In any case, people (animals) seek the optimal solution. However, there are limitations for optimization. One of these basic results is the NFLT. The problem of search and optimization, according to Ref.46, can be described using the fundamental matrix, whose columns are problems that need to be optimized and the strings are optimization strategies.

The essence of this theorem is that there is no optimization procedure that gives the best solution for all problems or even all of the examples of the particular problem. An example is the HOT (highly optimized tolerance) - the theory of complex systems which states that the system resistant to one disturbance can be fragile with respect to the others. There are many applications of NFLT to security, complexity, optimization, etc. ${ }^{[47]}$

Theorem establishes an equivalent level of optimization of algorithms by averaging the results of all problems. We can say that it is a black box optimization, i.e., "Optimization scenarios where one does not know the objective / fitness function to be optimized, but can only work with samples of that function". ${ }^{[47]}$

In particular, for optimization aprioristic knowledge plays a key role: ${ }^{[48]}$

If we have no prior knowledge about the problem (column) we are trying to optimize, then we must assume that all problems (columns) are equally likely, in which case, we cannot a priori expect any strategy (row) to perform any better than any other.

In other words, "there exists no strategy (regardless of its sophistication) that can outperform all others on all problems, or even on all instances of a particular problem, i.e., universal optimizers are impossible. Even humans, who sometimes seem to be capable of universal problem solving, are bound by the No Free Lunch Theorem".

The connection between the No Free Lunch Theorem and the problem of knowledge acquisition was described in an article. $^{[49]}$ In Ref.49, the author noted that if there was a universal algorithm for the acquisition of valuable new information, then this algorithm would contradict the No Free Lunch Theorem. The algorithm for the acquisition of new information could only work if the type of information the system encounters is known a priori. However, if the information is known, then this information is not new and is thus included in the system structure.

This contradiction was formulated by Melkikh ${ }^{[50]}$ earlier as the knowledge acquisition paradox. This paradox is the following:

If the information (image) is new, then it cannot be valuable (cannot be used) because it is not recognized. If the information (image) is recognized, the system has an etalon for it (which existed prior to its presentation), and, therefore, the information is not new. In both cases, the acquisition of knowledge does not occur. In this case, can the organism (artificial system) receive something new and useful? The scheme of recognition for this case is shown in Figure 1.

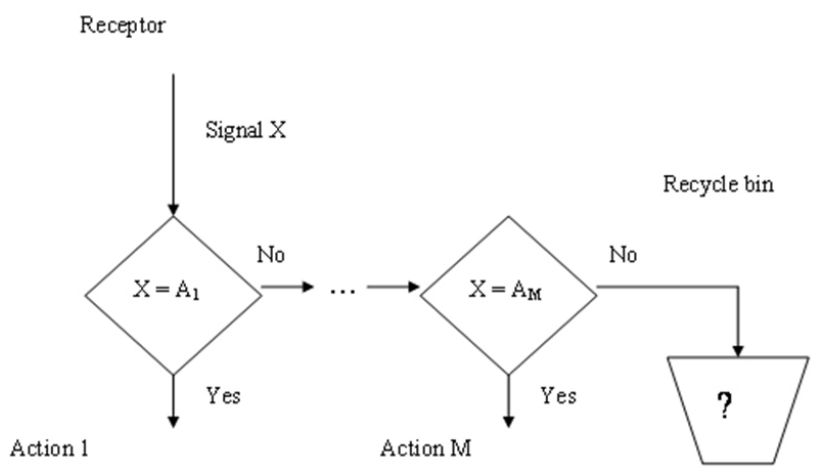

Figure 1: Scheme of the knowledge acquisition paradox. Receptor - is a device by which the system receives information about the environment; signal - is a physical change in the environment that is registered by receptor; etalon - is a standard with which the signal is compared when it is detected; recycle bin - is the storage of unnecessary information.

Melkikh $^{[50]}$ also considered a possible algorithm for creating new etalons for unrecognized images so that the system 
could perform adequate actions. This algorithm cannot begin its work because for this it needs to know a priori to which class an analyzed object belongs. This fact contradicts the original assertion that the object is not recognized.

We need a formal mathematical formulation of the paradox. However, we also need to find a connection between this paradox and the random search method, which is widely used in learning systems. Will the paradox retain its meaning in this case? This question will be considered below.

To prove the inconsistency of adjustment to an unrecognized situation, we construct a mathematical model of the process. Regardless of the nature of the processes, all of them can be divided into two classes: random and deterministic. Combinations of these two classes also exist. We consider deterministic processes in this section, and the role of stochastic processes in knowledge acquisition is discussed in section 5.

Consider the system (see Figure 2) with the following properties:

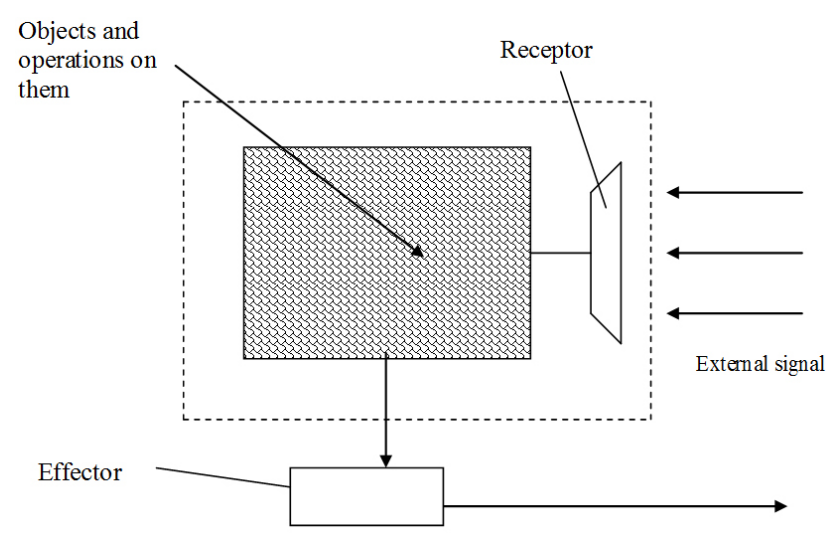

Figure 2: Scheme of the system structure

Property 1: The system contains internal independent objects (words of the language) $Q_{1} \cdots Q_{n}$. Operations $P_{1} \cdots P_{m}$ on objects are possible. Operations of new objects (operations) creation are not defined.

Property 2: The system has a receptor, which receives information about the state of the environment. The receptor is capable of detecting objects $q_{1} \cdots q_{m}$. In this case, the operations $p_{1} \cdots p_{k}$ can be performed on the objects. The receiving signal is compared with the internal objects present in the system (pattern recognition). The system compares the internal and external objects of the language. Mathematically, this comparison can be expressed in the form of equivalence (equality) of any internal object to some of the external objects.

Property 3: As a result of recognition (matching), some of the internal operations on objects trigger effectors; the system acts on the external environment. Every action is evaluated with the function $\Phi_{l}\left(Q_{i}, P_{i}\right)$ (gain). This value is greater when the system is more successful. Without loss of generality, we can assume that the system is designed so that there is a maximum condition: $\Phi_{l}\left(Q_{i}, P_{i}\right) \rightarrow \max$. The notion of "equivalence" is closely connected with the gain and represents adequate reflection of reality.

Property 4: The system has limited memory, which can store the results of previous measurements or intermediate operations.

As mentioned above, the operation of "preparation" will not be considered in the context of this problem. In this case, "preparation" refers to a physical effect on the organism (system) from the outside; as a result of this effect, objects and operations can be edited (deleted or created) (the system's physical structure may be changed). However, first, the operation can only be performed by another organism (system); second, this operation does not refer to the acquisition of knowledge (we need to consider the problem of learning in the larger system, including external system), and third, for living systems these operations are not known.

We must emphasize that the class of systems considered is very broad. This class includes both living and non-living systems. Indeed, any control system (even a non-intellectual system) uses this principle when receiving data. To further work with these data, the system should compare some reference value to the measured value. This is how all living organisms operate.

We consider the situation in which an image can be registered (measured) but not recognized. The system has no a priori information about the image.

Then recognition scheme can be depicted as follows (see Figure 3):

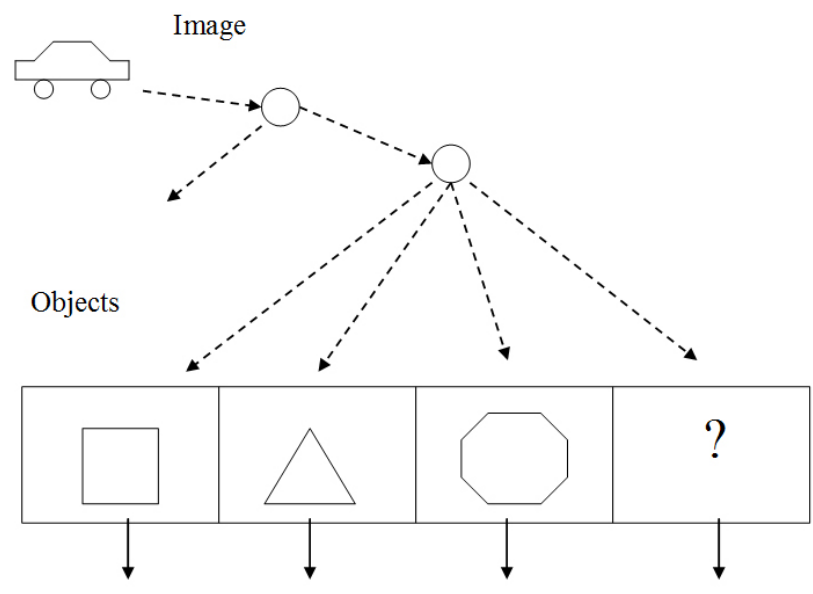

Operation $1 \quad$ Operation $2 \quad$ Operation 3

$?$

Figure 3: The recognition scheme

Let the image $\Omega$ appear to the organism (system) with the properties 1-4, which can be described in terms of the ex- 
ternal language of receptors (registered). However, no objects from the internal language are relevant (in the sense of equivalence) to external language objects, corresponding to this image. Then, the effector cannot perform operations and activities that would lead to the system obtaining a certain gain (payoff).

On the contrary, suppose that a universal translator from one language to another exists, which can work with an unrecognized image. However, in this case, this algorithm cannot be run because condition to run it cannot be formulated.

Suppose that the operation of supplementing the list of operations and objects with external alphabet phrases exists. However, this operation is controversial because it can be only performed if an external alphabet is part of the internal alphabet. If the phrase is part of the internal alphabet, the registered object is thus recognized, which contradicts the initial claim

From the point of view of pattern recognition and decisionmaking, the next version of the statement is controversial: "to create a new standard requires a new standard." If the image does not correspond to the internal standard, the system must create a new standard to make an adequate decision. However, its creation is self-contradictory because the algorithm that creates the standards must know them in advance. The following table summarizes the problem statement for the acquisition of knowledge in different branches of science.

Table 2: Knowledge acquisition problem

\begin{tabular}{|c|c|c|c|}
\hline & Recognition & Automata & Language \\
\hline $\begin{array}{l}\text { Properties of } \\
\text { the system }\end{array}$ & $\begin{array}{l}\text { System has } \\
\text { etalons }\end{array}$ & $\begin{array}{l}\text { Automaton with } \\
\text { equations is given }\end{array}$ & $\begin{array}{l}\text { Language } \\
\text { with rules } \\
\text { exists }\end{array}$ \\
\hline $\begin{array}{l}\text { Definition of } \\
\text { knowledge } \\
\text { acquisition }\end{array}$ & $\begin{array}{l}\text { Adequate } \\
\text { reaction to an } \\
\text { unrecognized } \\
\text { image }\end{array}$ & $\begin{array}{l}\text { A priori } \\
\text { determined gain in } \\
\text { an arbitrary } \\
\text { situation }\end{array}$ & $\begin{array}{l}\text { Inference of } \\
\text { new concepts } \\
\text { that have } \\
\text { semantic } \\
\text { meanings }\end{array}$ \\
\hline $\begin{array}{l}\text { Contradiction } \\
\text { of knowledge } \\
\text { acquisition }\end{array}$ & $\begin{array}{l}\text { An algorithm } \\
\text { that creates } \\
\text { new etalons is } \\
\text { contradictory } \\
\text { because it } \\
\text { cannot start } \\
\text { work }\end{array}$ & $\begin{array}{l}\text { In the equation of } \\
\text { state, this gain is } \\
\text { not defined. } \\
\text { Change in the } \\
\text { equation of state is } \\
\text { also not defined } \\
\text { (change of } \\
\text { structure cannot be } \\
\text { written in it) }\end{array}$ & $\begin{array}{l}\text { In an } \\
\text { axiomatic } \\
\text { system of } \\
\text { language, } \\
\text { creating new } \\
\text { concepts is not } \\
\text { defined }\end{array}$ \\
\hline
\end{tabular}

Thus, based on logical analysis of the recognition and decision-making by the system (organism), we reach the following conclusion: all programs of behavior can only be based on a priori defined internal objects and operations on these objects. Only innate behavior programs of living systems or a priori programs for technical systems are consistent. In turn, a system cannot acquire knowledge in the above sense (the synthesis of new behavior programs).

\subsection{Knowledge acquisition and learning automata}

M. L. Tsetlin was a founder of adaptive behavior modeling. He proposed to simulate the behavior of organisms based on the formalism of finite automata and coined the term "adaptive automaton".[51] A mathematical model of an adaptive automaton can be represented as follows:

- an automaton represents an object, capable in each time $t=1,2, \cdots$ to receive a limited quantity of signals $S \in\left(S_{1}, S_{2}, \cdots, S_{N}\right)$ and change depending on its internal state;

- an automaton can produce a limited quantity of actions $f \in\left(f_{1}, f_{2}, \cdots, f_{K}\right)$. The choice of action is determined by the internal state of the automaton;

- an automaton has a limited quantity of internal states $\varphi \in\left(\varphi_{1}, \varphi_{2}, \cdots, \varphi_{m}\right)$, where $\mathrm{m}$ is the memory capacity of the automaton.

It is assumed that the automaton is in some environment and that actions cause the automaton responses $\mathrm{s}$ for medium C. These reactions, in turn, are inputs to the automaton because the automaton uses these signals to decide on further actions. The simplest case is when all possible reactions of the environment are perceived by the automaton as belonging to one of two classes, the gain $(s=0)$ or loss $(s=$ 1). The appropriateness of the behavior of the automaton in some environment is to increase the number of favorable responses and to decrease the number of adverse responses.

The dynamics of the automaton are given by the equation $f(t)=F(\varphi(t))$ indicating the dependence of its action on its state $\varphi(t)$ and stochastic matrix $\left\|a_{i j}(s)\right\|, i, j=$ $1,2, \cdots, m, a_{i j}(s)$ is equal to the probability of transition from state $\varphi(t)=\varphi_{i}$ to state $\varphi(t+1)=\varphi_{j}$ under the influence of the input $s(t+1)$.

Determinate automata matrices $\left\|a_{i j}(s)\right\|$ consist of zeros and units. For two signals 0 and 1 , it is enough to determine two such matrices, $\left\|a_{i j}(0)\right\|$ and $\left\|a_{i j}(1)\right\|$.

In a stationary random environment, we can determine the expected value of a payoff, which is a measure of expedience of the behavior of the automaton.

The canonical equations for a deterministic automaton are as follows:

$\varphi(t+1)=\Phi(\varphi(t), s(t+1)), f(t)=F(\varphi(t))$

The first equation describes the change in the state of the automaton under the influence of the input variable $s$. The second equation describes the dependence of the actions of the automaton on its state. With respect to the behavior of the organisms, the first equation can be compared with changing of the nervous system (brain) of the organism under the influence of the information it receives from the environment. The meaning of the second equation is the modification of the behavior of the organism depending on its internal state. 
A stochastic automaton also has a finite number of states and a finite number of actions. Actions of the stochastic automaton are fully determined by its state $f(t)=F(\varphi(t))$ and the state matrix $\left\|a_{i j}(s)\right\|$ is stochastic, whereas $a_{i j}(s)$ is the probability of transition from the $\mathrm{i}$-th state to the $\mathrm{j}$-th state at a given input variable s. It is obvious that deterministic automata are special cases of stochastic automata.

A Markov chain describes the operation of the automaton in a stationary random environment.

The expected value $W(\Omega, C)$ of a payoff for automaton $\Omega$ in a random environment $\mathrm{C}$ is determined as the average of single payoffs. It is believed that the automaton has the appropriate behavior in environment $\mathrm{C}$ if the expected value of the payoff is larger than the payoff it will receive if it performs actions with equal probability regardless of the environmental states.

The simplest example of an adaptive strategy discussed in Ref.51 is that the automaton maintains the action at a payoff and changes the action at a loss. As a result of this strategy, the average payoff of the automaton increases.

Varshavskii and Vorontsova ${ }^{[52]}$ studied the behavior of stochastic automata with changing structures. Initially, the behavior of these automata is not appropriate, but upon receiving environmental reactions, these automata change their matrices and, consequently, the appropriateness of their behavior increases.

The structure of the automaton is given by the matrix $\left\|a_{i j}(s)\right\|$ that defines the state transitions $\varphi(t) \in$ $\left(\varphi_{1}, \varphi_{2}, \cdots, \varphi_{m}\right)$ for this or any other value of the input variable $s$, and the equations $f(t)=F(\varphi(t))$ define the action of the automaton depending on its condition.

In this case, the matrix of the state varies depending on the values of the input variable. Mechanisms for these changes may be different, but the state chosen from fixed and a priori defined sets of states is common. Completion of these sets through any mechanism is not determined. Accordingly, actions are also chosen from the set of possible actions. This behavior occurs when we are dealing with computer programs: it is only possible to choose the program of behavior from a priori existing programs.

In addition, if we consider that the automaton is found with a new situation that is a new feature of the environment, it is impossible to assert that the expectation of its payoff will be greater than any aprioristic value. Any gain from a particular action will significantly depend on information regarding the environment that is received by the automaton. Therefore, for unrecognized objects, gain cannot be determined, and it is impossible to draw any conclusion about the evidential effect of the new image on the behavior of the automaton.
What if this happens? Could an automaton acquire new knowledge?

The foregoing implies that to solve the problem of knowledge acquisition, the model of adaptive automata requires clarification.

Learning automata represent a further development of the concept of adaptive automata. ${ }^{[16,18]}$ In general, an advantage of modeling learning using the theory of automata is that the structure and operation of the automaton is completely defined mathematically. Challenges to the idea of learning in relation to automata are definitely not associated with the acquisition of knowledge. Indeed, because the state of the automaton and its internal alphabet are completely axiomatically (a priori) defined and not subject to completion or revision because the appropriate mechanisms are not provided, the acquisition of new knowledge in this system is impossible. The typical behavior of this automaton can be characterized as an adaptation to random environments. This applies to automata with variable structure ${ }^{[52]}$ because the changes apply only to the probabilities of various transitions and not the structure itself.

To investigate the possibility of artificial systems acquiring knowledge, we use the language of learning automata ${ }^{[16,18]}$ as well as mathematical models of adaptive behavior. ${ }^{[53]}$ However, as mentioned above, because the learning automaton is fully formalized and the new state is not defined, after the receiving of an unknown image this automaton will not work. Therefore, we can unambiguously conclude that based on the theory of learning, the acquisition of knowledge by automata is impossible. However, because this problem occurs in the simulation of artificial life, it is necessary to expand the model of the learning automaton.

As an extension of the model, consider the following mechanisms by which the structure of the automaton can be changed: the creation of new actions and states by external operations such that it can make another automaton, the creation of new actions and states for the automaton by a universal algorithm of knowledge acquisition, and the creation of new actions and states for the automaton by a random search or trial and error.

Based on the above, we consider a formal model for the process of knowledge acquisition as follows:

$$
\begin{aligned}
& \varphi(t+1)=\Phi(\varphi(t), s(t+1)) \\
& f(t)=F(\varphi(t))+\xi(t) \\
& s(t)=R(c(t)) \\
& \varphi(k+1)=\Omega(\varphi(t), c(t))
\end{aligned}
$$




$$
\varphi(k+1)=U(\varphi(t), s(t))
$$

The first equation describes the change in the state of the automaton under the influence of the input variable $s$. Now, however, the input variable is not a state of the environment but only the result of its recognition. The second equation describes the dependence of the actions of the automaton on its state. In this equation, $\xi(t)$ is a random process.

The third equation describes the process of recognition and represents the dependence of the input variable on the state of environment. It may be that for some cases, the value $\mathrm{c}=\mathrm{c}^{*}$ (unrecognized image) does not correspond to any value of $s$. The fourth equation describes the generation of new actions by another automaton $\Omega$ in a particular situation that arises in the environment. It is assumed that automaton $\Omega$ has its own structure and recognizes the state of the environment. For brevity, the equations describing the behavior of automaton $\Omega$ have been omitted. The fifth equation describes the generation of new actions by the automaton through a universal algorithm.

As a result, at each step the automaton wins $W_{i}$ which characterizes the adequacy of the automaton, and the automaton action program is structured to maximize this benefit.

Note that the proposed system of equations is the most common and does not have any restrictions in the description of the known behavior of live and artificial systems.

The structure of the automaton is shown schematically in Figure 4.

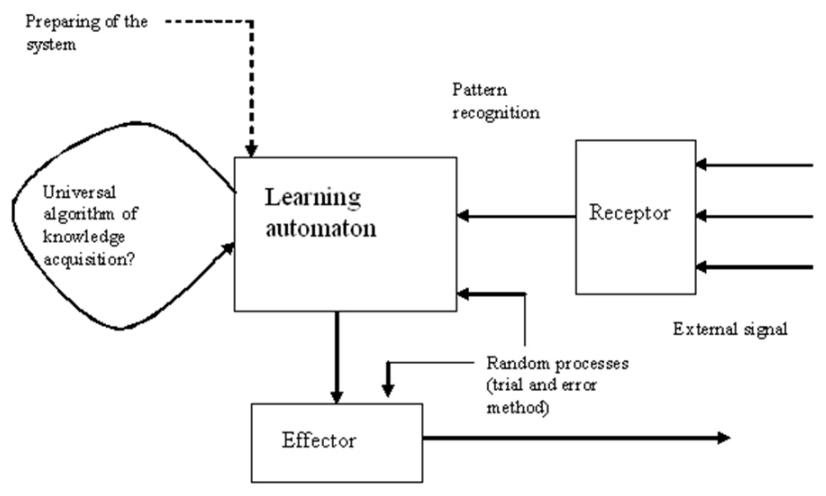

Figure 4: Structure of the learning automaton

We now define the acquisition of knowledge in accordance with the proposed model.

Definition. This will be understood by the acquisition of knowledge of such a process, followed by the emergence of an environmentally unrecognized image $\mathrm{c}^{*}$, not including preparation of the systems and only to provide the system information regarding the external environment through receptors, which results in a behavior of the system that is estimated to increase the expectation of winning at some preset value of $W_{0}: W\left(c^{*}\right)>W_{0}$.

The assignment of the image to unrecognized objects indicates that there is no prior information about the object.

Consider the first possible mechanism for knowledge acquisition, the existence of a universal algorithm. As noted above, the existence of a universal algorithm is refuted on the basis of the No Free Lunch Theorem. Therefore, the action $\varphi_{k+1}$ in the mathematical model is not defined, a priori it does not match the payoff and it is impossible to assume it will be either adequate or lead to payoffs. If this action is defined, then the function $\varphi_{k+1}$ pre-exists in a list, which was not used previously. This option leads us to an automaton with a variable structure similar to that discussed above. As previously noted, this automaton can learn but cannot acquire knowledge.

The second possibility of knowledge acquisition by an artificial organism is related to its preparation or the change of its structure due to an outside action. This possibility was considered earlier. For external automata, there is a function that changes the structure of other automata, and the conditions for this function and the possibility of newly created properties of the automaton are also defined. Does this partially solve the problem of knowledge acquisition? On the one hand, as demonstrated earlier, these processes occur very often in the preparation of computing and intelligent systems and do not refer to learning, much less to knowledge acquisition. On the other hand, both the automata can be regarded as a single automaton, and for this single automaton, we can re-formulate the task of knowledge acquisition. However, the problem in this case is reduced to the original problem in that consideration of the entire system only infinitely postpones the problem.

\subsection{Hypothesis of innate behavior}

Thus, we conclude that new behavior programs will not occur as a result of learning. The animal cannot adequately respond to an unrecognized object. This conclusion leads to the need for a new paradigm of animal behavior. We state the main ideas of this paradigm:

(1) Innate programs of behavior are formed on the basis of genes during ontogenesis (morphogenesis).

The process of formation of these programs is not obvious because, for example, in an organism, there is clearly a discrepancy between the information contained in the genes and complexity of the nervous system. However, the modeling of morphogenesis is a separate problem and is not considered in this paper.

(2) As a result of receptors receiving signals from the environment, remembering and recognition occur.

Memory of the time or spatial distribution of the signals used by the organism to run a priori behavior programs is the most adequate for the observed situation. If recognition is not possible (the appeared 
image is new), then either no a priori programs start or the programs that correspond most closely (on the tree of recognition) to the recognized image are run.

(3) At the presence of uncertainty in the environment or errors of receptors aprioristic programs run only after several repetitions.

Repetition can reduce random error in the registration signal. This situation is largely similar to the decrease of random error in measurements.

(4) Another response to the uncertainty of the environment is a trial and error method, which allows the system to select the most appropriate aprioristic behavior program.

(5) An interaction with another organism that transfers the experience of one organism with the environment (see items 2-4) to another is only possible if both the organisms have the same a priori programs relating to the present situation.

(6) High forms of behavior are contradictory. In particular, the system cannot create a new concept that is adequate for the new situation.

(7) All programs of animal behavior are innate. For technical systems, they are aprioristic programs.

Thus, animal learning caused by uncertainty in the environment represents the method of choice for the a priori (innate) program, which is most appropriate for a certain situation. The new behavior program cannot be formed on the basis of experience or interaction with other animals.

On the basis of the allegations made, we consider that, paradoxically, the human (higher animals) is able to adequately respond to images, which he and his ancestors have never seen. How could such an ability arise from the process of evolution? What does this ability represent?

How is this consistent, for example, with statements such as "monkey learn sign language" (not to mention humans), etc.? Can we assume that such language is innate? Can we assume that any human language is innate?

To answer this and other questions, we discuss the features of items 1-7 of the hypothesis in more detail and consider possible experiments to verify our claims.

\section{Discussion}

\subsection{Can new behavior programs arise from random processes?}

We have examined the deterministic processes of animal behavior and showed that the emergence of new behavior programs in such processes is contradictory. Now, we consider random processes and show that the contradiction remains.

We consider algorithms of system actions when the recorded image is not recognized (see Figure 5). One common algo- rithm is a random search algorithm, which is widespread in animal behavior. In the proposed model, an algorithm is modeled using a random walk in the space of possible system structures (automata). However, this raises the question of how many systems exist, i.e., the dimension of the search space. If the search space is unbounded, then the solution could require infinite time. If the space is limited and the a priori condition of the search is specified, then this program can only be aprioristic. During the search among the possible objects and operations, the set in which the search is conducted must first be defined. If the search set is not defined, then the work may be broken during a search of the system. This result will occur because there will be occasional combinations of system elements in which the response to an external signal is inadequate.

The search can occur based on a brute force algorithm or chance. Randomness, in turn, can be created artificially (dynamic chaos, random number generator) or have a natural origin (thermal noise). However, this randomness is not sufficient. There should be an enumeration algorithm of system structures (machines); a set of possible system structures (machines) must be defined. Additionally, there must be a set of criteria to end the search. This condition may be either to achieve a predetermined gain or comparison (and memorizing) of all received gains; the strategy choice corresponds to the maximum.

In this case, the behavior is innate. The contradiction of creating new programs remains regardless of how they were created (randomly or deterministically). New programs cannot be created that are proven to be good in some sense. The variants cannot obtain a guaranteed win (more gain than was pre-assigned).

This "controlled noise" is of type A behavior.

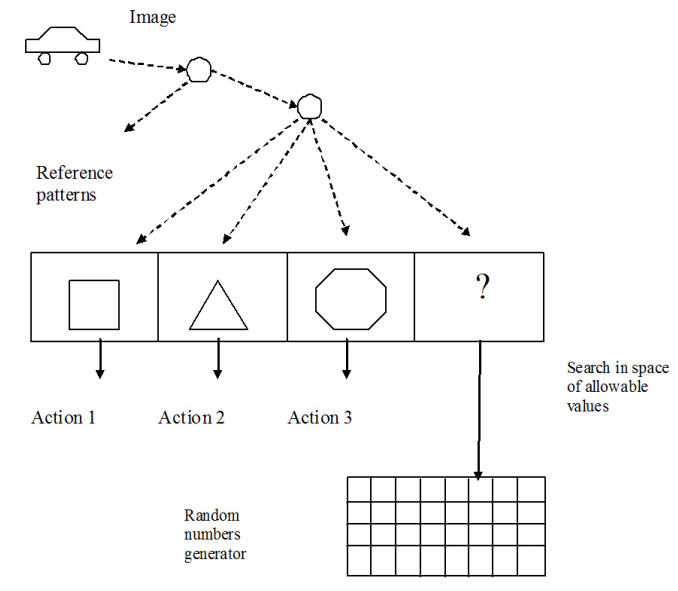

Figure 5: Unrecognized image processing

We show that with a priori information, the solution lies in a bounded set. In this case, the system (automaton) does not perform acquisition of knowledge (behavior of type B), but 
learning (behavior of type A). Moreover, restrictions on the set, which allow it to enumerate in real time, also mean that, in this situation, learning, but not the acquisition of knowledge occurs. The application of the trial and error method is impossible without a property that requires a priori information about the object being analyzed. This result contradicts the definition of knowledge acquisition given above.

To ensure that any restriction of the search area is associated with a priori information, we use classical information theory.

We consider the trial and error method as a series of experiments, which will result in finding an adequate algorithm or the absence of an adequate algorithm. In information theory, the degree of uncertainty of experiment $\beta$ with possible outcomes and their probabilities $p_{1}, p_{2} \cdots p_{k}$ is usually characterized by the Shannon entropy:

$$
H(\beta)=-\sum_{i=1}^{k} p_{i} \log _{2} p_{i}
$$

If this value equals zero, the outcome of the experiment is known in advance. Any measurement or supervision $\alpha$ prior to experiment $\beta$ can limit the quantity of possible experimental outcomes and thus reduce a degree of its uncertainty. The fact that realization of experiment $\alpha$ reduces a degree of uncertainty in experiment $\beta$ is reflected in the fact that the conditional entropy $H(\beta / \alpha)$ of experiment $\beta$ under the condition of $\alpha$ is less than (more precisely - less than or equal to) the initial entropy of the same experiment. Thus, if experiment $\beta$ does not depend on $\alpha$, realization of $\alpha$ does not reduce the entropy of $\beta$ :

$$
H(\beta / \alpha)=H(\beta)
$$

If the result of $\alpha$ completely predetermines an outcome of $\beta$, the entropy decreases to zero: $H(\beta / \alpha)=0$. Thus, the difference

$$
I(\alpha, \beta)=H(\beta)-H(\beta / \alpha)
$$

specifies how much realization of experiment $\alpha$ reduces the uncertainty of experiment $\beta$. This difference represents the quantity of information in experiment $\beta$, which is contained in experiment $\alpha$. Here, information (according to the information theory) is understood as the uncertainty which is removed when a message is received.

There is an interrelation between the number of experiments and information received as a result of each experiment. For example, if the experience is found with an adequate algorithm, each experience corresponds to receiving one bit of information. Suppose that the system has $n$ degrees of freedom, each of which may be in one of $m$ states. If, at the beginning of the experiment, there is no a priori information to find an adequate algorithm, then we obtain the average number of steps required to achieve it, a number equal to the total number of possible states:

$$
N=m^{n}
$$

If $n$ is sufficiently large, then the number of steps is exponentially large even for $m=2$.

The number of steps corresponds to Shannon entropy:

$$
H=\log _{2} N=n \log _{2} m
$$

In this case, each bit of a priori information reduces the search area in half.

If the number of experiments is reduced by using an algorithm compared to the entire enumeration, the a priori information in the system is present. This information should be included in the algorithm used. In this case, the acquisition of knowledge is absent because there is a priori information about the object.

In reply to a question on whether there is a general solution in this set, we can argue the following in the same way: without a priori information about the existence of solutions in this area, no other search method, except brute force, can exist. In this case, the enumeration will occur in all possible automata (as permitted by the laws of nature and the laws on the environment). Naturally, the power of this set is exponentially large.

As an example, consider the solution to the problem of password cracking. Imagine that an unrecognized image is a code (an information sequence). Then, the random search of code variations (an attempt to break the code), implemented by the automaton, will correspond to the trial and error method. This sequence of actions is committed by replacing letters, which changes the code as follows: AABCFDDD $\rightarrow$ AABCFFDD. Any such action will be viewed as an experiment, which makes the automaton. The experiment ends when the sequence coincides with a target sequence that is not known in advance. The challenge is to determine the minimum number of steps required to achieve the first sequence and find the optimal search algorithm.

This problem is one of the classic problems of cryptography; researchers $^{[54]}$ proved that in the absence of a priori information, the problem is NP-hard, i.e., the problem requires an exponentially large number of steps for the solution.

In which case the problem of cracking the password can be solved using some quicker method (for example, retaining the correct letters and changing the wrong ones)? Obviously, this method can be only used if a priori information about the environment exists (in this case, the sequence). Indeed, to use this method, the sequence should be prepared in a special way: there must be a mechanism that defines whether a letter is "good" or not. This mechanism must exist for each letter individually, not only for the entire sequence 
as a whole. The enumerating region is significantly limited, which implies the existence of a priori information about the environment.

If such a "cumulative search" works under any conditions (i.e., without any a priori constraints), then the task of cracking the password would not be a NP-hard; in general this class of problems would not exist. However, the problem $\mathrm{NP} \neq \mathrm{P}$ is one of the Millennium problems, and this problem has still not been solved. ${ }^{[55]}$

This, of course, does not mean that the trial and error method is not effective; this method is effective under certain conditions. The effectiveness of the random-search method has been proven for automata with variable and other methods, such as genetic algorithms, swarm intelligence, and Monte Carlo, among others.

Finally, we note that if the trial and error method can be regarded as a universal algorithm for knowledge acquisition, then such a statement contradicts the No Free Lunch Theorem. Thus, for a system (living or artificial), algorithms exist to gain knowledge using the trial and error method; the algorithm requires a priori information about the environment in which the machine exists. This result contradicts the definition of knowledge acquisition, which distinguishes this concept from learning.

If, however, we summarize the results of the analysis of other algorithms to obtain new knowledge of an organism (artificial system), we conclude the following: all these algorithms either lead to contradictions or do not lead to acquisition of knowledge (behavior of type B) but instead represent a type of learning (type A behavior).

Thus, a random search triggered innate programs. The search only serves to determine which of these programs is the most suitable for the conditions - this is the essence and purpose of learning. The search occurs in a small (nonexponential) number of variants.

Thus, each animal must have an innate program of trial and error (such as genetic algorithms or other soft computing). The result of this program will be the ranking of other congenital programs according to their relevance to the situation. Genetic predetermination of the program is confirmed by experiments. ${ }^{[56]}$

Although knowledge acquisition is impossible the system (alive or artificial) can still obtain information as a result of some its actions. As a result of experience (communication with the environment), the system receives the information (or removes uncertainty) that one of the inherent behavior programs is suitable in this case.

\subsection{Randomness and repetition as a method for de- creasing errors}

As noted above, conditioned reflexes and other behaviors often require the repetition of certain actions. How can we justify such repetition? Many beneficial programs are created such that they will only run after some preliminary steps. The simplest of these actions can be repetition. This repetition is a direct consequence of the uncertainty and error occurring in the environment.

When modeling animal behavior, researchers usually use the utility function (fitness). ${ }^{[57,58]}$ We show that in the simplest case, repetition leads to a reduction of costs.

Errors occur each time the device measures the environment, leading to the possibility of erroneous recognition. Therefore, the larger is the measurement error, the smaller is the fitness $\Phi$ (fitness is at a maximum if errors are absent). We shall label the maximum fitness as $\Phi_{0}$. Then, we can write the following (if the errors are relatively small):

$$
\Phi=\Phi_{0}-\Delta
$$

where $\Delta$ is a positive value denoting the decrease of fitness resulting from a measurement error. From error theory, we know that random error decreases during repeated measurements as the number of measurements increases (if a systematic inaccuracy is present, it can be included in the maximum fitness). However, each measurement requires energy, and, therefore, the fitness will decrease. We shall label the decrease of fitness caused by the energy consumption for one measurement as $\varepsilon$. Then, we have the following for the fitness:

$$
\Phi=\Phi_{0}-\varepsilon n-\frac{\Delta}{\sqrt{n}}
$$

where $n$ is the number of measurements.

The factor $1 / \sqrt{n}$ appears because of the decrease of the random error during repeated measurements. This formula can be written for the case when the measurement time is small compared to the characteristic behavior time. Alternatively, when measurements are slow, the fitness will decrease because the organism will not have time to measure quick changes of the environment.

The fitness (1) may have an extremum with respect to $n$ :

$$
\frac{\mathrm{d} \Phi}{\mathrm{d} n}=-\varepsilon-\frac{1}{2} \frac{\Delta}{n^{3 / 2}}=0
$$

Then, for $n$, we have:

$$
\begin{aligned}
& n=\left(\frac{\Delta}{2 \varepsilon}\right)^{2 / 3} \\
& \Phi(X, Y)=\Phi_{0}(X, Y)-\varepsilon^{1 / 3} \Delta^{1 / 3}\left(\frac{1}{2^{2 / 3}}+2^{1 / 3}\right)
\end{aligned}
$$


Because the number of measurements is an integer, the extremum exists only at $\Delta / \varepsilon>2$. Therefore, an organism, which is in a complicated environment, may find it unfavorable to immediately change its behavior as the environment is altered, but instead might prefer to perform a set of measurements and only then begin to act. In other words, to start some available programs, an organism should first recognize its environment and determine the necessary programs.

This behavior explains the fact that, for example, living organisms form conditioned reflexes most often as a result of recurrence of an external signal. Thus, repeated measurements of the state of the environment by an organism decrease the error during operation of aprioristic programs.

\section{Continual computations and parallel search.}

There are other models of computation, ${ }^{[59]}$ such as continual calculations. The main feature of continual computations is that the variables of the system are continuous. However, we can easily show that regardless of the computing model, NP-hard problems remain. Despite the fact that the continuous systems formally have an infinite number of degrees of freedom, they do not easily solve complex problems. There are two cases: 1) a computer system, composed of agents, each of which is controlled separately or 2) a computer system, composed of agents, each of which individually uncontrollable.

An example of the second case is a continuous medium (fluid) or the electrical potential of a cell membrane. In this case, the system can contain any number of particles (agents), but the amount of information it can carry is finite. This fundamental conclusion was made by Shannon. ${ }^{[60]}$ The same conclusion applies, of course, to the calculations. This conclusion connects with the fact that a digital signal is necessary at some stage. In this case, ease of transmission and processing of continuous signals is not due to rapid processing of information (and certainly not with the solution of NP-hard problems), but rather due to the fact that in some lines of communication, transmitting these signals is convenient.

The second limiting case (1) means that each agent is able to perform calculations. Then, a large collection of such agents will be able to perform parallel computing. However, this result does not mean that all NP-hard problems are automatically resolved. Unlike the case of (2), each agent must be properly organized; its behavior should be governed by certain signals from the outside (it must consume energy, etc.). In fact, the behavior creates a large computer system. The larger is the computer system, the harder is the problem to be solved. For such a system to be able to solve hard problems (e.g., pattern recognition), the system (as well as any other computer system) should have standards (etalons). At the same time, the paradox stated above remains. Moreover, its formulation does not depend on the specific implementation of the physics of the computing processes.
Statements by Ivanitskii ${ }^{[61,62]}$ that the paradox of knowledge acquisition in living systems has a simple solution, are incorrect. According to Ref.61 and 62, the brain is a biochemical analogous machine, which is based on principles that are different from the principles of operation of computers, learning automata and other devices. One of the principles the author considers is the principle of competition for resources and energy. The mechanism of neural networks does not involve enumeration of all possible variants, but is based on auto-wave principles of the interaction of the collective modes.

However, we note that competition for resources is not a new principle, peculiar only to living systems. The existence of such competition by itself does not lead to new logical principles.

Ivanitskii argues that the existence of neuronal clusters large dynamic collections of neurons - by themselves can avoid enumeration of a large number of variants, which automatically leads to the acquisition of knowledge.

We also note that parallel processing (parallel search) will only lead to a polynomial reduction in the number of steps and, therefore, does not solve the problem of searching an exponentially large space. Indeed, if there are two parallel working automata, then they can divide the search area in half. In this case, the average seek time (in the absence of prior information) will be halved. In the case of $k$ automata, when using parallel computing to solve the problem, the average time of its solution will be $k$ times smaller than the time for one automaton. Because the number of automata participating in the parallel solution is always a polynomial (the number of neurons, the number of processors), then we can easily see that such a parallel solution will not lead to significant progress in the case of exponentially large numbers that characterize the search space.

\subsection{Social learning, intelligence and knowledge ac- quisition}

Social learning is often considered separately because of its complexity and importance to complex communities. Social learning is often considered an important part of the learning experience as a transfer from parent to offspring. ${ }^{[5]}$ Numerous experimental data confirm this conclusion. However, in terms of the problem addressed in this article, social learning is not fundamentally different from other types of learning. We consider the case when an organism receives new information from its parents or other animals. Can the organism adapt itself to new conditions in this case? Considering what has been said above, the answer is no.

Importantly, we note that in this case, the organism also receives information via receptors. Therefore, any other organism (a member of the flock, the parent, etc.) is interpreted to be part of the environment. Consequently, the aforementioned scheme measurement - recognition - deci- 
sion making holds. Whichever actions the parent makes, they can be adequately interpreted only in one case: when the signal has been recognized (compared with a standard sample). In turn, this comparison is only possible if the standard sample has already been available in the organism (has been congenital).

What is the role of the experience transfer between animals? This role obviously reduces to triggering of aprioristic (congenital) behavior programs. Indeed, if the environment has uncertainties, an important question is which programs to start. The start of behavior programs that are not adequate to changes in the environment can be catastrophic for an organism. Thus, social learning allows ranking of innate behavior programs for their relevance in the social environment.

Note also that "supervised learning" in animals is fundamentally different from the "supervised learning" in neural networks. The difference is that a neural network "teacher" has the ability to prepare the system (that is, to set the connections weights between neurons form the outside), and for animals it is impossible. All acts of the "teachers" can be perceived only through pattern recognition.

The analysis of human learning is a separate problem, but analysis based on formal rules does not change the inconsistency of knowledge acquisition. Various researchers ${ }^{[63-65]}$ ) have suggested that some of the concepts of human language are congenital. In this case, however, the formation mechanism of the remaining concepts is unclear.

For humans, we often use the term "intelligence" to differentiate from the cognitive abilities of animals. However, we need a more precise definition of intelligence. If we associate intelligence with the notion of self, "I", or other internal characteristics, the definition is almost untestable and subjective. Arranging an experiment to confirm the feeling of "I" in animals is difficult. The concept of "intelligence" is the ability to logically link acquired knowledge. Some derivatives of this ability include the ability to create new concepts and the ability to respond adequately to the new environment. However, the algorithm of creating new concepts can only work if this new concept is defined a priori. Accordingly, we need to discuss the formation of concepts and the fact that these aprioristic concepts are effective under certain conditions. "Intelligence" in this situation means many a priori programs are adapted to essentially different situations.

\subsection{Where innate programs of behavior can stored?}

The paradox is not due to the conjecture that all behavior programs are innate (though this statement is also important and requires special consideration). Above all, the paradox arises when considering where to store innate (a priori) information. If there were a universal algorithm for knowledge acquisition, storage in relatively short information sequences, such as genes, would be possible. If (as shown

Published by Sciedu Press above), there is no such algorithm, the data should be stored in the genes (or their analogs in artificial systems). However, the information capacity of genes is not sufficiently large. This result is particularly evident for higher animals and humans, whose genome sizes are approximately of the order of $10^{9}$ bits. Attempts to construct artificial systems that can navigate in a complex situation (recognize patterns and make decisions) show that this system requires a memory size many orders of magnitude larger. For example, humans and animals freely operate visual images for computer processing, which would require many megabytes of memory.

How can we resolve the paradox of too much innate information?

We can assume that living systems have a large amount of hidden innate information not related to genes. One such opportunity for the storage of large amounts of information is provided by quantum mechanics. As is well known the quantum particles that represent q-bits can store more information than their classical analogs. The main property of q-bit is that it may exist not only in one of its two states, but also in both of them simultaneously. This property allows for the parallel operations that are used in quantum computers. Can quantum entanglement characterize biologically important molecules (proteins, DNA, RNA)? A number of papers are devoted to this subject. For example, Davies $^{[66]}$ examined the role of quantum mechanics in life processes. In particular, Davies noted that the uncertainty principle limits the precision of a quantum clock (Wigner inequality). The same logic can be applied to the process of protein folding. The experimental dependence of folding on the length of the protein is consistent with the theoretically derived dependence. Some articles suggest quantum models of intracellular metabolism, ${ }^{[67]}$ intracellular processes and storage. ${ }^{[68,69]}$ Models of quantum information processing in neurons ${ }^{[70,71]}$ are also presented.

A number of scientists believe that the possible quantum behavior of biologically important molecules allows us to discuss the quantum basis of intelligence. ${ }^{[72-74]}$

If the proteins within cells are in entangled states and this state can be maintained for a long time by some mechanism (which can be tested experimentally), this quantum entanglement can serve as an important information resource for the organization of animal behavior. This additional resource can solve the paradox of the large amount of inherent information that is necessary for the implementation of innate behavior programs. Therefore, information can be stored in the entangled quantum states of proteins and not primarily in genes where there is not enough space. From the point of view of language, this information represents a large and hidden portion of the overall information. Thus, there are no controversies when some properties of the higher levels of the system are not related to the prop- 
erties of the lower levels of the system. Some authors ${ }^{[43]}$ use the term "system properties" or "emergent", but during the ontogenesis of an organism, all properties need to be determined. When the physical transition from a molecular to macroscopic system or organism occurs, the transition properties of the organism at higher levels must be a consequence of the properties of the lower levels.

Based on the theory of dynamical systems, any measurement is always associated with irreversibility and randomness. By contrast, the Copenhagen interpretation of quantum mechanics, associate collapse of the wave function with measurement, such as the interaction of a quantum particle with a macroscopic device. However, the definition of the "device" is uncertain; therefore, it would be logical to associate the ideas of "device" and "measurement" with irreversibility and chaos. Then, the situation in which the wave function collapses becomes quite certain.

Melkikh $^{[75,76]}$ proposed that some of the coherent (characterized by a wave function, which is the solution of the Schrödinger equation) quantum states of the intracellular molecules are abnormally stable. "It can be assumed that the structure of biological molecules involves additional selection rules for quantum particles, which significantly slow decoherence. It is possible that coherent quantum states and entanglement of quantum states arise even in the synthesis of proteins (and DNA and RNA) and are then stored and maintained at the expense of non-local interactions".

In terms of the issues addressed in this study, it can be assumed that the properties of biological molecules are as follows: the quantum states of biologically important molecules, such as proteins in neurons, can store an exponentially large amount of information.

Therefore, the information contained in DNA that is necessary for protein synthesis may be much less than that contained in its entangled quantum states. We can say that this information is a library that is used for problem solving.

However, the question is, from where do these quantum states for animal behaviors arise? Currently, the answer to this question cannot be obtained experimentally, but it can be assumed that the structure of proteins, which is due to the properties of the electrons in the atoms, already contains the information. The idea of the special properties of elementary particles has been discussed previously (see, for example, the anthropic principle ${ }^{[74]}$ ).

\subsection{Possible experiments to test the hypothesis on innate behavior programs}

Is it possible to design an experiment to obtain a definitive answer to the question of whether all programs of behavior are innate?

As noted above, ${ }^{[78]}$ current experiments cannot accurately identify the chain of elementary events that occur during progression from genes to behavior. This is due to the complexity of living systems and the fact that it is difficult to perform a non-invasive experiment on the organism and receive enough information.

To understand whether the organism is obtaining something novel, data are needed regarding as to what is innate and what is not with respect to the organism. However, this is not a trivial matter because inherent information cannot manifest itself under certain influences. For example, when we work with computers, we know that certain programs manifest themselves or run only under very specific conditions. It is now recognized that the relationship between genes and behavior is complex. There is a significant difference between the statement "as a result of switching off this gene, certain types of behavior disappear (modified)" and the statement "the gene contains the entire program necessary to implement this type of behavior." The first statement can be verified relatively easily, although difficulties can arise. ${ }^{[78]}$ Experiments to test the second statement are quite complex but possible in principle.

What experimental requirements are necessary to identify the presence of innate behavior programs? Obviously, such an experiment would allow us to follow the entire decisionmaking chain at the molecular level such that a change in the environment $\rightarrow$ recognition process $\rightarrow$ decision-making $\rightarrow$ appropriate action. The most valuable experiment is one that would reveal the appropriate response of the animal to a stimulus that was not adjusted either during past generations or over the course of evolution. Therefore, it is necessary to artificially create a new situation that is unpredictable from the point of view of the animal. This primarily refers to a rapidly changing environment, which is related to the evolution of society, ecosystems, and technology during recent decades and centuries. For example, an appropriate response of the animal to synthetic chemicals, which did not previously exist in nature, may occur. Additionally, training a higher animal in properties of human behavior and problem solving may also occur. It is necessary to identify the response of each neuron and, if possible, to resolve all the reactions, including changes in the proteins.

Another direction for experimental research is to determine where large amounts of information that are required for complex behaviors, such as the recognition of complex images and smells, are stored. This can be accomplished by identifying the molecular structures of the decision-making chain.

Therefore, the purpose of these experiments is to simultaneously measure the states of the proteins involved in learning and decision making.

Experimental methods for the identification of molecular changes that occur in the nervous system include luminescence and X-ray analysis. Currently, luminescence is widely used to determine the state and position of macromolecules, 
such as proteins. In this case, the problem lies in recording the state of many proteins in many cells simultaneously, such as during the formation of new synaptic contacts. This is a technically challenging but feasible task.

$\mathrm{X}$-ray analysis techniques, (such as X-ray Free Electron Laser) are advantageous because they can provide high resolution up to a single atom. Identifying the position of a large number of atoms or molecules is also difficult but feasible in principle.

The use of such methods will allow for the development of neurogenetics analyses at the atomic level. What conclusions can be drawn from these experiments?

First, we will obtain an understanding of the ability of each organism to adapt to the environment. Second, for areas such as synthetic biology and artificial life, it is important to understand the general laws of artificial systems that allow these systems to adapt to unforeseen circumstances. Therefore, our principle conclusion is that is impossible to create a self-learning synthetic system. With respect to artificial life, this suggests that the behavior programs of these organisms can only be aprioristic or previously created. Third, our conclusion imposes restrictions on a wide range of intelligent systems that are used in various fields of engineering and human activities.

\section{Conclusions}

A model of learning in animals that is based on the neurogenetics of behavior and on the theory of complexity and pattern recognition is proposed in this study, and we also consider the analogy between artificial intelligence algorithms and the properties of living systems. Pavlovian condition- ing that is related to the task of clustering is demonstrated. Additionally, we show that Pavlovian conditioning can be implemented in the absence of the recognition of new objects. However, more complex forms of learning are contradictory, and the paradox of learning is formulated, which has the following double meaning: higher animals and humans can adequately respond to a situation that could not be innately programmed, but general algorithms to adapt to unknown situations are contradictory, and if these new situations exist as innate programs, it is unclear where such programs can be stored due to the huge amount of information required for these programs.

Therefore, a large part of behavior, such as learning by trial and error, is consistently explained by innate programs. Another aspect of behavior, such as the creation of concepts and insight, is usually explained by abstract learning abilities. However, these abilities are contradictory. The proposed hypothesis is that this aspect of behavior is also based on innate programs. In both cases, however, there is a problem regarding storage of the large amount of information that is required for these behaviors.

An important conclusion for synthetic biology and artificial intelligence is that it is impossible to build a self-learning artificial organism or intelligent system. The hypothesis that animal behavior is based on innate behavior programs is proposed in this study. The quantum entanglement states of proteins may allow for coding, which allows for the storage of much more information. This information will be a hidden part of the internal language, and structures within these states may be inherent, which is a consequence of the structure of the atoms and molecules. Experiments to test these hypotheses are also proposed.

\section{References}

[1] Waddell S, Quinn WG. Flies, genes, and learning. Ann Rev Neurosci. 2001; 24: 1283-309. PMid:11520934 http://dx.doi.org /10.1146/annurev. neuro.24.1.1283

[2] Wiener N. Cybernetics or Control and Communication in the Animal and the Machine. MIT Press; 1948.

[3] Bloch E, Cardon S, Iberall A, Jacobowitz D, Kornacker K, Lipetz L, McCulloch W, Urquhart J, Weinberg M, Yates F. Introduction in biological systems science. NASA, Washington; 1971.

[4] Anokhin PK. Biology and neurophysiology of the conditioned reflex and its role in adaptive behavior. Pergamon Press, New York; 1974.

[5] Shettleworth SJ. Cognition, Evolution, and Behavior (2nd Ed). Oxford Univ. Press; 2010.

[6] Thorpe WH. Learning and instinct in animals, 2nd edn, Methuen, London; 1963.

[7] Meyer J-A, Wilson SW. (Eds) From animals to animats. Proceedings of the First International Conference on Simulation of Adaptive Behavior. The MIT Press: Cambridge, Massachusetts, London, England; 1990.
[8] Donnart JY, Meyer JA. Learning Reactive and Planning Rules in a Motivationally Autonomous Animat. IEEE Transactions on Systems, Man, and Cybernetics - Part B: Cybernetics. 1996; 26(3): 381-395. PMid:18263041 http://dx.doi .org/10.1109/3477. 499790

[9] Tsitolovsky LE. A model of motivation with chaotic neuronal dynamics. Journal of Biological Systems. 1997; 5(2): 301-323. http: //dx.doi.org/10.1142/S0218339097000199

[10] Edelman G. Neural Darwinism. Oxford University Press, New York; 1989.

[11] Edelman G, Tononi G. Consciousness. How matter becomes imagination. Penguin Books, London; 2000.

[12] Maninng A, Dawkins MS. Animal Behavior. 5-th Edition. Cambridge University Press; 1998.

[13] Bloom FE, Lazerson A, Hofstadter L. Brain, mind, and behavior. Freeman, New York; 1988.

[14] Simon HA. Why should machines learn? In RS Michalski, J CRarbonell, TM Mitchell(Eds), Machine learning: an artificial intelligence approach. San Mateo: Morgan Kauffmann; 1983.

[15] Nadin M. Not everything we know we learned. In M. Butz et al. (Eds.): Anticipatory Behavior in Adaptive Learning Systems. Foun- 
dations, Theories, and Systems, LNAI. 2684, Berlin. Heidelberg. NewYork: Springer-Verlag; 2003. pp 23-43

[16] Narendra KS, Thathachar MAL. Learning Automata: An Introduction, Englewood Cliffs, NJ. Prentice Hall; 1989.

[17] Marsh C, Gordon TJ, Wu QH. Stochastic optimal control of active vehicle suspensions using learning automata, Proceedings I Mech Eng Part I, Journal of Systems and Control Engineering. 1993; 207: 143-152.

[18] Narendra KS, Thathachar MAL. Learning Automata. A Survey. IEEE Transactions in Systems, Man and Cybernetics. 1974; 4(4): 323-334.

[19] Valiant LG. A theory of the learnable. Communications of the ACM. 1984; 27: 1134-1142. http://dx.doi.org/10.1145/1968.19 72

[20] Garey M, Johnson D. Computers and Intractability: A Guide to the Theory of NP-Completeness. Freeman, San Francisco; 1979.

[21] Luger GF. Artificial intelligence. Structures and strategies for complex problem solving. Fourth edition. Addison Wesley; 2003.

[22] Luger GF. Cognitive Science: The Science of Intelligent Systems. Academic Press, San Diego and New York; 1994.

[23] Minsky ML The Society of Mind. William Heinemann Ltd, London; 1987.

[24] Vilhelm C, Ravaux P, Calvelo D, Jaborska A, Chambrin M-C, Boniface M. Think!: a unified numerical-symbolic knowledge representation scheme and reasoning system. Artificial Intelligence. 2000; 116 (1-2): 67-85.

[25] Kamsu-Foguem B, Tchuenté-Foguem G, Allart L, Zennir Y, Vilhelm Y, Mehdaoui H, Zitouni D, Hubert H, Lemdani M, Ravaux P. User-centered visual analysis using a hybrid reasoning architecture for intensive care units. Decision Support Systems. 2012; 54 (1): 496-509. http://dx.doi.org/10.1016/j.dss.2012.06.009

[26] d'Avila Garcez AS, Lamb LC, Gabbay DM. Neural-Symbolic Cognitive Reasoning, Cognitive Technologies. Springer-Verlag, Berlin Heidelberg; 2009.

[27] Brachman RJ. Systems that know what they're doing. IEEE Intelligent systems. 2002; 6: 67-71. http://dx.doi.org/10.1109/M IS. 2002.1134363

[28] Salakhutdinov R, Tenenbaum J, Torralba A. One-shot learning with a hierarchical nonparametric Bayesian model. Technical Report. 2010; MIT-CSAIL-TR-2010-052.

[29] Ohsuga SA. Consideration to knowledge representation - an information theoretic view. Bulletin of Informatics and Cybernetics.1984; 21(1-2): 121-135.

[30] Gottfredson LS. Mainstream Science on Intelligence (editorial). Intelligence. 1997; 24: 13-23.

[31] Ohsuga SA. Intelligence for Upgrading Information. In N. Zhong et al (Eds). Web Intelligence Meets Brain Informatics. LNAI 4845; 2007. pp 97-121.

[32] Pavlov IP. Conditioned reflexes: an investigation of the physiological activity of the cerebral complex. Oxford University Press. London; 1927.

[33] Bouton ME. Learning and Behavior: A Contemporary Synthesis. Sunderland, MA: Sinauer; 2007

[34] Rescorla RA, Wagner AR. A theory of Pavlovian conditioning: Variations in the effectiveness of reinforcement and nonreinforcement, Classical Conditioning II, A.H. Black \& W.F. Prokasy, Eds., Appleton-Century-Crofts; 1972. pp 64-99.

[35] Miller RR, Barnet RC, Grahame NJ. Assessment of the RescorlaWagner Model. Psychological Bulletin (American Psychological Association). 1995; 117 (3): 363-386. PMid:7777644

[36] Cattell RB. The description of personality: Basic traits resolved into clusters. Journal of Abnormal and Social Psychology. 1943; 38 : 476-506. http://dx.doi.org/10.1037/h0054116

[37] Jain AK, Murty MN, Flynn PJ. Data clustering: a review. ACM Computing Surveys. 1999; 31: 264-323. http://dx.doi.org/1 $0.1145 / 331499.331504$

[38] Duda RO, Hart PE, Stork DG. Pattern classification. 2nd ed., John Wiley \& Sons, N.Y.; 2001.
[39] Hong YY. Learning Assignment Order of Instances for the Constrained K-Means Clustering Algorithm. IEEE Transaction on System, Man and Cybernetics, Part B. 2009; 39 2: 568574. PMid:19109091 http://dx.doi.org/10.1109/TSMCB.2 008.2006641

[40] Agrawal AA. Phenotypic Plasticity in the Interactions and Evolution of Species. Science. 2001; 294: 321-326. PMid:11598291 http://dx.doi.org/10.1126/science.1060701

[41] Whitman DW, Agrawal A. What is phenotypic plasticity and why is it important? Phenotypic Plasticity of Insects: Mechanisms and Consequences (ed. by D. W. Whitman and T. N. Ananthakrishnan), Science Publishers, Enfield, New Hampshire; 2009. pp 1-63.

[42] Glanzman DL. Common Mechanisms of Synaptic Plasticity in Vertebrates and Invertebrates. Minireview. Curr Biol. 2010; 20: 3136. PMid:20152143 http://dx.doi.org/10.1016/j.cub. 200 9.10 .023

[43] Noble D. The Music of Life. Biology Beyond Genes, Oxford University Press, Oxford; 2006.

[44] Searle JR. Is the brain's mind a computer program? Sci Am. 1990; 1: 26-31.

[45] Gorlich D, Artmann S, Dittrich P. Cells as semantic systems. Bioch Bioph Acta. 2011; 1810: 914-923. PMid:21569823 http://dx.d oi.org/10.1016/j.bbagen.2011.04.004

[46] Wolpert DH, Macready WG. No Free Lunch Theorems for optimization. IEEE Trans Evol Comput.1997; 1: 67-82. http://dx.doi.o $\mathrm{rg} / 10.1109 / 4235.585893$

[47] Wolpert DH, Macready WG. Coevolutionary free lunches. IEEE Transactions on evolutionary computation. 2005; 9: 721-735. http: //dx.doi.org/10.1109/TEVC.2005.856205

[48] Ho YC, Pepyne DL. Simple explanation of the No-Free-Lunch Theorem and its implications. J Optim Theory Appl. 2002; 115: 549570. http://dx.doi.org/10.1023/A:1021251113462

[49] Melkikh AV. First principles of probability theory and some paradoxes in modern biology (comment on "21st century: what is life from the perspective of physics?" by G.R. Ivanitskii). Phys Usp. 2011; 54(4): 449-451.

[50] Melkikh AV. Can Organism Pick up New Valuable Information from the Environment? Biophysics (Biofizika). 2002; 47(6): 1053-1058.

[51] Tsetlin ML. Finite automata and models of simple forms of behavior. Russian Mathematical Surveys. 1963; 18(4): 1-27. http: //dx.doi.org/10.1070/RM1963v018n04ABEH001139

[52] Varshavskii VI, Vorontsova IP. On the behaviour of stochastic automata with variable structure. Automation and Remote Control. 1963; 24: 327-333.

[53] Butz M, Sigaud O, Gerard P. Internal models and anticipations in adaptive learning systems. In M. Butz et al. (Eds.): Anticipatory Behavior in Adaptive Learning Systems. Foundations, Theories, and Systems, LNAI. 2684, Berlin. Heidelberg. NewYork: SpringerVerlag; 2003. pp 89-109.

[54] Mao W. Modern cryptography: theory and practice. Prentice Hall, Professional Technical Reference; 2003.

[55] Aaronson S. NP-complete problems and physical reality. ACM SIGACT News, complexity theory column. 2005; 36(1): 30-52. http://dx.doi.org/10.1145/1052796.1052804

[56] Klein TA, Neumann J, Reuter M, Henning J, Yves von Cramon D, Ullsperger M. Genetically Determined Differences in Learning from Errors. Science. 2007; 318: 1642-1645. PMid:18063800 http://dx.doi.org/10.1126/science.1145044

[57] McFarland D. Animal behavior. Pitman, University of Oxford; 1985.

[58] McFarland D, Houston A. Quantitative ethology: The state space approach. Pitman Advanced Pub Program, Boston; 1981.

[59] Simeonov PL. Integral biomathics: A post-Newtonian view into the logos of bios. Prog Bioph Mol Biol. 2010; 102 2-3: 85-121.

[60] Shannon CA. Mathematical Theory of Communication, Bell System Technical Journal.1948; 27: 379-423, 623-656.

[61] Ivanitskii GR. 21st century: what is life from the perspective of physics? Physics - Usp. 2010; 53(4): 327-356. http://dx.doi . org/10.3367/UFNe.0180.201004a.0337 
[62] Ivanitskii GR. Remembering a random choice kills alternatives (reply to comment on ,21st century: what is life from the perspective of physics?"). Physics - Usp. 2011; 54(4): 451-454. http: //dx.doi.org/10.3367/UFNr.0181.201104p.0451

[63] Osherson D, Stob M, Weinstein S. Systems that learn. MIT Press. Cambridge MA; 1986.

[64] Pask G. The cybernetics of human learning and performance. Hutchinson; 1975.

[65] Fodor JA. The Modularity of Mind: an Essay of Faculty Psychology. MIT Press; 1983.

[66] Davies P. Does quantum mechanics play a non-trivial role in life? BioSystems. 2004; 78: 69-79. PMid:15555759 http://dx.doi.o rg/10.1016/j.biosystems. 2004.07.001

[67] Davies P, Demetrius LA, Tuszynski JA. Implications of quantum metabolism and natural selection for the origin of cancer cells and tumor progression. AIP ADVANCES. 2012; 2: 011101. PMid:22489276 http://dx.doi.org/10.1063/1.3697850

[68] Matsuno K. Cell motility as an entangled quantum coherence. BioSystems.1999; 51: 15-19. http://dx.doi.org/10.1016/S 0303-2647 (99) 00009-X

[69] Igamberdiev AU. Quantum computation, non-demolition measurements, and reflective control in living systems. Biosystems. 2004; 77: 47-56. PMid:15527945 http://dx.doi.org/10.1016/j.b iosystems . 2004.04.001

[70] Beck F, Eccles JC. Quantum aspects of brain activity and the role of consciousness. PNAS.1992; 89: 11357-11361. http://dx.doi.o rg/10.1073/pnas.89.23.11357
[71] Hameroff S. Conduction pathways in microtubules, biological quantum computation and consciousness. Biosystems.2003; 64: 149168. http://dx.doi.org/10.1016/S0303-2647(01)00183-6

[72] Georgiev D. Consciousness operates beyond the timescale for discerning time intervals: implications for Q-mind theories and analysis of quantum decoherence in brain. NeuroQuantology. 2004; 2: $122-145$.

[73] Melkikh AV. Congenital programs of the behavior as the unique basis of the brain activity. NeuroQuantology. 2005; 2: 134-148.

[74] Melkikh AV. Congenital programs of the behavior and nontrivial quantum effects in the neurons work. BioSystems. 2014; 119: 1019. PMid:24704210 http://dx.doi.org/10.1016/j.biosyst ems. 2014.03.005

[75] Melkikh AV. Biological complexity, quantum coherent states and the problem of efficient transmission of information inside a cell. BioSystems. 2013; 111: 190-198. PMid:23438638 http://dx.d oi.org/10.1016/j.biosystems.2013.02.005

[76] Melkikh AV. Quantum information and the problem of mechanisms of biological evolution. BioSystems. 2014; 115: 3345. PMid:24184874 http://dx.doi.org/10.1016/j.biosyst ems. 2013.10.005

[77] Carter B. Large Number Coincidences and the Anthropic Principle in Cosmology. IAU Symposium 63: Confrontation of Cosmological Theories with Observational Data. Dordrecht: Reidel; 1974. pp 291-298.

[78] Glanzman DL. Behavioral neuroscience: no easy path from genes to cognition. Curr Biol. 2012; 22 9: 302-304. http://dx.doi.o $\mathrm{rg} / 10.1016 / \mathrm{j}$.cub.2012.03.034 